九州大学学術情報リポジトリ

Kyushu University Institutional Repository

\title{
STATISTICAL METHOD OF DETERMINING A CUT OFF VALUE BETWEEN NORMAL AND DISEASE GROUPS
}

Kim, Inyoung

Department of Applied Statistics, Yonsei University

Choi, Yeon-ho

Department of Applied Statistics, Yonsei University

Chung, Hyun Cheol

Department of Applied Statistics, Yonsei University

Rha, Sun Young

Department of Applied Statistics, Yonsei University

他

https://doi.org/10.5109/12579

出版情報: Bulletin of informatics and cybernetics. 36, pp.63-72, 2004-12. Research Association of Statistical Sciences

バージョン :

権利関係 : 


\title{
STATISTICAL METHOD OF DETERMINING A CUT OFF VALUE BETWEEN NORMAL AND DISEASE GROUPS
}

\author{
by \\ Inyoung Kıм, Yeon-ho Сног, Hyun Cheol Chung, Sun Young Rha \\ and \\ Byung Soo KIM
}

Reprinted from the Bulletin of Informatics and Cybernetics

Research Association of Statistical Sciences, Vol.36
FUKUOKA, JAPAN
2004




\title{
STATISTICAL METHOD OF DETERMINING A CUT OFF VALUE BETWEEN NORMAL AND DISEASE GROUPS
}

\author{
By \\ Inyoung Kıм, \\ and \\ Byung Soo KIM
}

\begin{abstract}
The classical method to determine the cut off value between normal and disease group is to calculate two standard deviations of the difference between mean values of two groups under the independence assumption. However, this independence assumption does not hold in general, and in our study in particular, when two biomarker proteins of breast cancer are measured several times for a few years. In this paper we propose a method to determine the cut off value for this case by implementing the inherent nature of the study using linear mixed effect model. We use a linear mixed effect model to take it into consideration that the subject is of a random effect. Furthermore, we can also estimate the growth curve of the biomarker values as time elapses. For a fixed type I error rate we calculate the conditional probability that the test is positive given that the subject does have the disease and then compare the sensitivity of our method with that of classical method using a leave-one-out cross validation. We observe that our method is more efficient than the classical method.
\end{abstract}

Key Words and Phrases: Biomarker, Cross validation, Cut off value, ELISA, Linear mixed effect model.

\section{Introduction}

Biomarkers have been searched for in various cancers although quite a few of them have been known. In our study we find two biomarker proteins, say Protein 1 and Protein 2, from breast tissues which can be detected using Enzyme-Linked Immunosorbent Assay (ELISA) in the blood of the subject (Rha et al., 2000). It is also important to determine the cut off value between the disease and the normal groups once the biomarker proteins

\footnotetext{
* Department of Applied Statistics, Yonsei University, 134 Shinchon-dong, Seodaemun-gu, Seoul, 120749 tel +82-2-2123-4541, bskim@yonsei.ac.kr

$\dagger$ Department of Applied Statistics, Yonsei University, 134 Shinchon-dong, Seodaemun-gu, Seoul, 120749 tel $+82-2-2123-4541$

¥ Department of Applied Statistics, Yonsei University, 134 Shinchon-dong, Seodaemun-gu, Seoul, 120749 tel $+82-2-2123-4541$

$\S$ Department of Applied Statistics, Yonsei University, 134 Shinchon-dong, Seodaemun-gu, Seoul, 120749 tel +82-2-2123-4541

ฯ Department of Applied Statistics, Yonsei University, 134 Shinchon-dong, Seodaemun-gu, Seoul, 120749 tel $+82-2-2123-4541$
} 


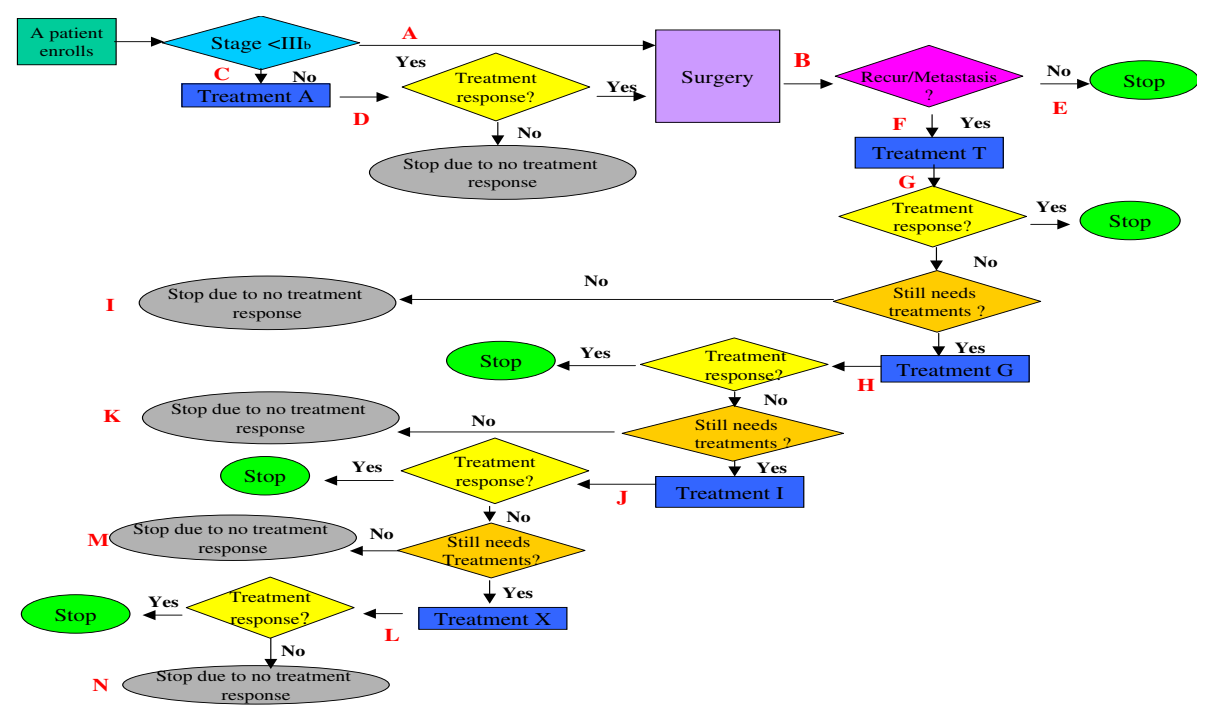

Figure 1: The chart of examinations to a monochrome type. The flow chart of subject starts at Status A or C. Whenever diagnosis indicates "no cancer" or "no treament response" at a certain Status, the flow chart of subject will stop at that Status. Otherwise, it will proceed along the chart.

have been developed. The classical method to determine the cut off value is to calculate two standard deviations from the difference between mean values of two groups under the independence assumption (Rha et al, 2000). However, this method is not appropriate when the assumption is violated. In our study Protein 1 and Protein 2 of each subject are measured several times for a few years by following a flow chart of the examination which is shown in Figure 1. Thus, observations of each subject are not independent.

The purpose of this paper is to propose a statistical method of determining a cut off value between normal and disease groups when observations are not independent. We develop the method to determine the cut off value using a linear mixed effect model which allows us to model that the subject is of a random effect. The computational methods are described in Pinheiro and Bates (2000) and we follow the general framework of Lindstrom and Bates (1988). The model formulation is described in Laird and Ware (1982). We calculate the false positive probability after we fixed the probability of type I error at a low level. We compare the false positive probability of our method with that of the classical method using leave-one-out cross validation. Furthermore, we estimate the growth curve of the biomarker values as time elapses as we proceed along the flow chart of examination of Figure 1. We also determine what factors affect the biomarker values such as sex, age, treatment and surgery of which the problem the classical method can not formulate. Hence, our method utilizes more information of data and furthermore, it does not require the independence assumption. 


\section{Materials and methods}

We collected 54 female patients who had breast cancers, 40 female subjects and 13 male subjects from normal group in Cancer Metastasis Research Center (CMRC), College of Medicine, Yonsei University, Korea. An ELISA was used to measure two biomarkers from blood of subjects. For each subject we obtain measurments of two proteins by following the flow chart of the examinations in Figure 1. The subject starts at stages $\mathrm{A}$ or $\mathrm{C}$ in the flow chart. Whenever the subject is diagnosed having no cancer at a certain stage, the flow chart of the subject will stop at that stage. Otherwise, it will proceed along the flow chart. Fourteen out of 54 patients are measured once; some patients took a surgery at the first examination and then were diagnosed having no cancer. For each of these patients we have only one measurment on the biomarker. Other patients were diagnosed as "no need of treatment" at the first examination becasue their cancer stages were beyond the treatment. These patients also had one measurment on the biomarker. In general the number of measurments depend on the Status of disease and situation of each patient. We also have age information on each subject. The histograms of two biomarkers between the normal and the disease groups are given in Figure 2 .

\section{Statistical Methods}

The biomarker values of some subjets are measured several times. We use a linear mixed effect model (LME) considering the subject as a random effect. We include following variables in the model; age, the length of time in terms of the month between the first examination and current examination, disease Status, whether the surgery is performed, and whether treatment is made. Finally, we fitted the following linear mixed effect model of equation (1) to describe the biomarker value $y_{i j k l m n}$ measured on the $n$ month interval between the first and the current examinations at the $m$ th surgery indicator of the $l$ th treatment indicator at the age when the $k$ th measurement was made for the $j$ th subject in the $i$ th group:

$$
\begin{aligned}
y_{i j k l m n} & =\beta_{0}+\beta_{1} \operatorname{Group}_{i}+\operatorname{Subj}_{j}+\beta_{2} \operatorname{Age}_{k}+\beta_{3} \operatorname{Trt}_{l}+\beta_{4} \operatorname{Sur}_{m}+\beta_{5} \operatorname{Month}_{n} \\
& +\beta_{6}(\operatorname{Sur} * \text { Month })_{m n}+\epsilon_{i j k l m n},
\end{aligned}
$$

where $\operatorname{Subj}_{j} \sim N\left(0, \sigma_{1}^{2}\right), \epsilon_{i j k l m n} \sim N\left(0, \sigma_{i}^{2}\right), i=1$ (normal group), 2 (disease group), $j=1,2, \ldots, N_{i}, k=1,2, \ldots, L_{i j}, l=1$ (no treatment), 2 (treatment), $m=1$ (before surgery), 2 (after surgery), $n=1, \ldots, L_{i j}$.

The fixed effects are group, age, surgery, treatment, time (in terms of month) interval and interaction between the surgery and the time interval. The $\mathrm{Subj}_{j}$ denotes the subject random effect, assumed to be independent for different subject, and the within group errors $\epsilon_{i j k l m n}$ are independent and identically distributed, assumed to be independent of the random effect.

We checked graphically on assumptions of the linear mixed effects model (plots not shown). First, a plot of the standardized residual versus the fitted values showed that the residuals were distributed symmetrically around zero with an approximately constant variance. It did not indicate the violation of the constant variance assumption of the within-group error. Second, the normal probability plot of the standardized residual indicated a little violation of the normality assumption for the within-group errors. The assumption that the subject followed a normal distribution with a constant variance did 

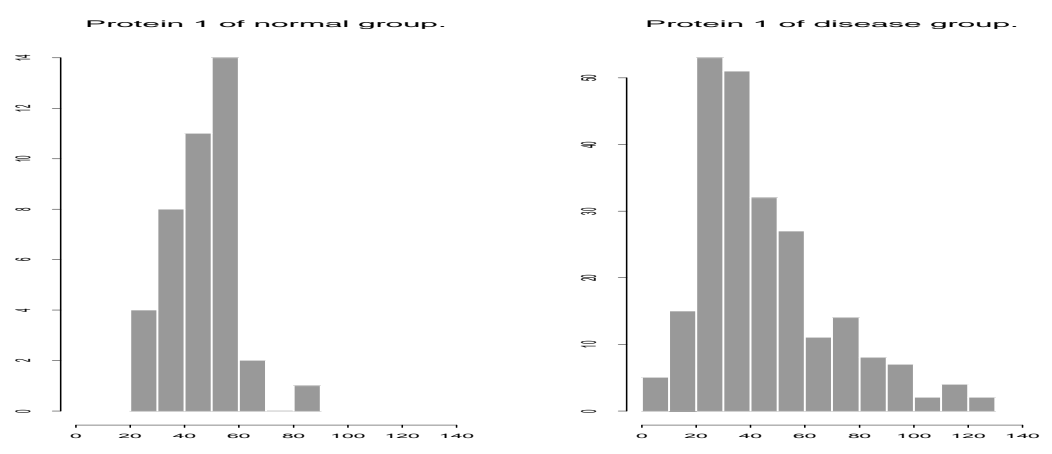

(a)
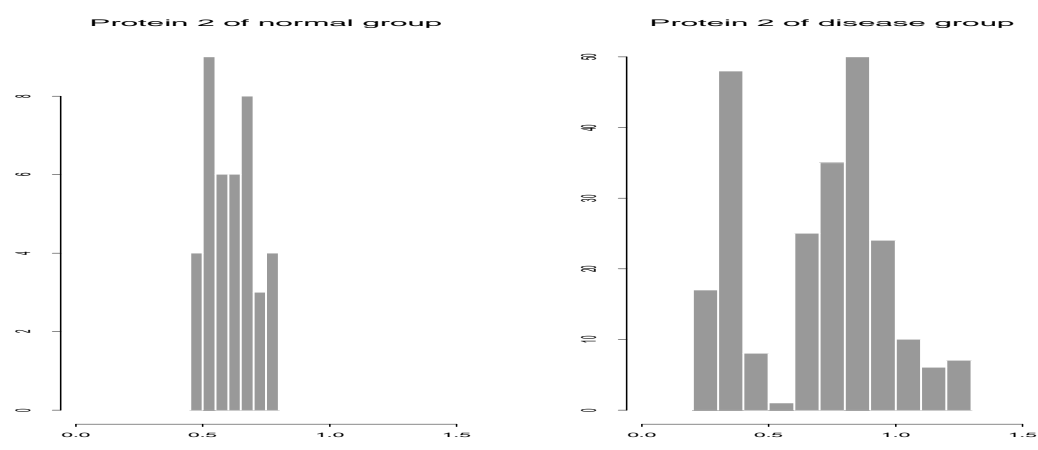

(b)

Figure 2: Histograms of Protein 1 (a) and Protein 2 (b) between the normal (left) and the disease (right) groups. 
not look violated much. Finally, the adequacy of the fitted model was better visualized by fitted curves on real data which was shown in Figure 3. These plots showed that the linear mixed effect model of equation (1) fitted the data quite well. The data were spreaded around fitted curve due to the variance of observations on each person. The fitted curve represents the mean of observation of each person.

Furthermore, we subgrouped the disease group by following the flow chart of Figure 1. Stages of patients were divided into five stages, i.e., I, II, $\mathrm{III}_{a}, \mathrm{III}_{b}$ and IV when a patient enrolled. we considered whether the stage of a patient was more serious than stage $\mathrm{III}_{a}$. In Figure 1, the flow chart starts at Status A or C. Status A stands for the indication of a cancer with stage less serious than IIIb which we denote using a symbol "Stage $<\mathrm{III}_{b}$ ". Status B is the notation for the case when a patient has a surgery. Status C means that a patient has cancer with stage IIIb or IV. Status D denotes the case when a patient receives treatment A after Status C. Status E represents the indication of no cancer after surgery. Status F stands for the indication of cancer after surgery. Status $\mathrm{G}$ implies that a patient receives treatment $\mathrm{T}$ after Status F. Status H means the case when a patient has treatment G. Status I denotes the indication of no cancer after Status G. Status J represents the case when a patient takes treatment I after Status H. Status $\mathrm{K}$ indicates no treatment response after Status H. Status L represents the case when a patient takes the treatment X after Status J. Status M denotes no treatment response after Status J. Status N stands for no treatment response after Status L. Therefore, the flow chart of subject will stop at certain Status whenever diagnosis indicates "no cancer" or "no treatment response". Otherwise, it will proceed along the chart.

First, we consider two subgroups; one consists of the Status $\{\mathrm{B}, \mathrm{E}, \mathrm{I}\}$ corresponding to diagnosis of having no cancer and the other group, $\Omega-\{\mathrm{B}, \mathrm{E}, \mathrm{I}\}$, where $\Omega$ denotes all the Status in Figure 1. Second, we also consider three subgroups; the first one is composed of Status $\{\mathrm{A}, \mathrm{C}, \mathrm{D}\}$ in disease group, the second one is $\{\mathrm{B}, \mathrm{E}, \mathrm{I}\}$, and the third one consists of $\Omega-\{\mathrm{A}, \mathrm{B}, \mathrm{C}, \mathrm{D}, \mathrm{E}, \mathrm{I}\}$. We replace the group indicator with the subgroup indicator in the model (1) and then fit the model to compare each of these subgroups with the normal group.

\section{Results}

\subsection{Comparison of biomarker values between male and female in the nor- mal group}

We first test whether the biomarker values between female and male are different using t-test and Wilcoxon rank sum test. We found that there was a significant difference between male and female in both tests whose results were given in Table 1. This result imlpies that the cut off values between males and females should be seperately determined.

\subsection{Age effect}

We also investigate whether the age affects the biomarker values between the disease group and the normal group. Since the patient group consists of all female, we only consider female subjects. There is no evidence that age affects the biomarker values between two groups, of which detailed results are presented in Table 2. 
Table 1: Test result whether the biomarker value between female and male is different using t-test and Wilcoxon rank sum test.

\begin{tabular}{|c|cc||cc|}
\hline Biomarker & \multicolumn{2}{|c||}{ Protein 1 } & & Protein 2 \\
\hline Test & $\mathrm{t}$ & Wilcoxon rank sum & $\mathrm{t}$ & Wilcoxon rank sum \\
\hline t stat & 6.367 & 5.470 & -5.917 & -4.979 \\
p-value & 0 & 0 & 0 & 0 \\
\hline
\end{tabular}

Table 2: Test results whether age affects the biomarker values between the disease and the normal groups.

\begin{tabular}{|c|c|c|c|c|}
\hline Biomarker & Mean & S.E & t-value & p-value \\
\hline Protein 1 & -0.266 & 0.243 & -1.091 & 0.276 \\
Protein 2 & 0.003 & 0.002 & 1.282 & 0.201 \\
\hline
\end{tabular}

\subsection{Linear Mixed Effect Model Fit}

After we fit the linear mixed effect model including the group indicator(the disease and the normal groups), the time (in terms of month) interval, the surgery indicator, the treatment indicator, and the interaction between the time interval and the surgery and considering subjects as a random effect, we found the evidence of the difference of Protein 1 between the disease and the normal groups. We also detect the difference of Protein 1 between without treatment and with treatment groups. Furthermore, we observe that the growth curve of Protein 1 between before surgery and after surgery has changed as time elapses( Figure 1). We also note the difference of Protein 1 between before surgery and after surgery. On the other hand, there is no evidence of the difference in Protein 2 between the disease and the normal groups. We also detect no difference in Protein 2 between without treatment and with treatment groups. However, there is an evidence of the difference of Protein 2 between before surgery and after surgery groups. The growth curve of Protein 2 between before surgery and after surgery groups has changed as time elapses, as is indicated in Figure 3.

The test results are given in Table 3 . These results imply that Protein 1 is more sensitive to the development of disease than Protien 2.

\subsection{Comparisons of each of subgroups with normal group}

First, we fit the model to compare the normal group(N) with two subgroups which are defined in the previous section. As a result of fit, there is no evidence of the difference of Protein 1 between two subgroups. We did not find the difference of Protein 1 between the normal group and the subgroup $1,\{\mathrm{~B}, \mathrm{E}, \mathrm{I}\}$. However, there is an evidence of the difference of Protein 1 between the normal group and the subgroup 2, $\Omega-\{\mathrm{B}, \mathrm{E}, \mathrm{I}\}$. As we expect from the result of previous section, there is an evidence of the surgery effect and the treatment effect. We also notice that the growth curve of protein 1 between before surgery and after surgery groups has changed as time elapses. We did not find no difference of each protein between two subgroups and no difference between the normal 

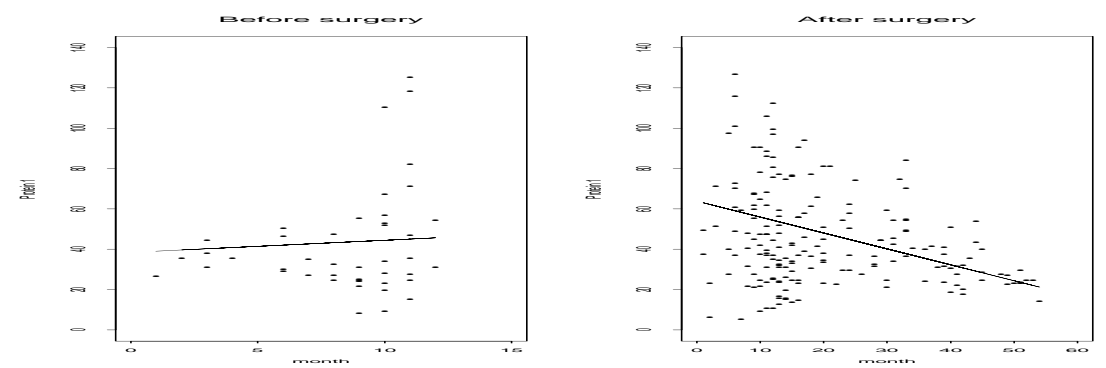

(a)
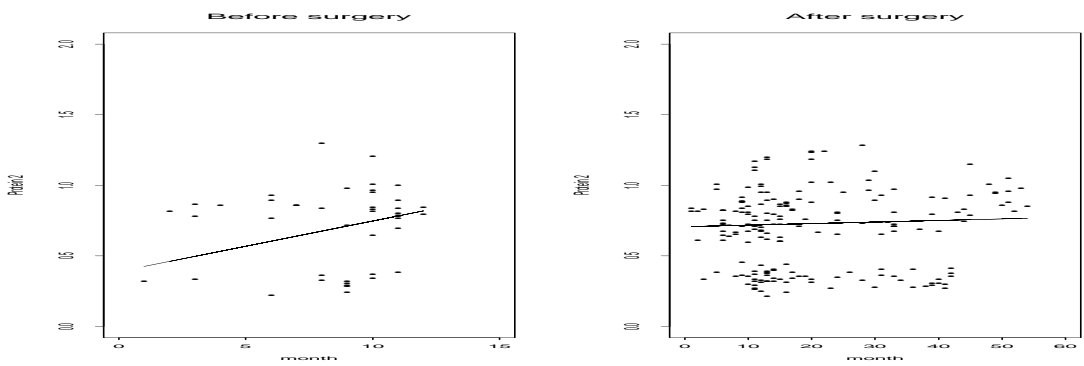

(b)

Figure 3: A linear mixed effect model fits of Protein 1 (a) and Protein 2 (b) between before surgery (left) and after surgery (right). These plots show that the linear mixed effect model fits quite well our data which are spreaded around the fitted curve due to the variance of observations of each person. The fitted curve represents the mean of observations of each person. 
Table 3: Significance test results by fitting a linear mixed effect model which included for covariates the group indicator(disease vs normal groups), the time (in terms of month) interval, the surgery indicator, the treatment indicator and the interaction between the surgery and the time interval.

\begin{tabular}{|c|cccc||cccc|}
\hline Covariate & \multicolumn{3}{|c||}{ Protein 1 } & \multicolumn{3}{c|}{ Protein 2 } \\
\hline & Mean & S.E. & t stat & p-value & Mean & S.E. & t stat & p-value \\
Group & 7.125 & 2.533 & 2.812 & 0.0060 & -0.026 & 0.041 & -0.640 & 0.524 \\
Month & -0.801 & 0.106 & -7.500 & 0.0001 & 0.009 & 0.003 & 2.527 & 0.012 \\
Treat & -5.661 & 1.158 & -4.885 & 0.0001 & -0.011 & 0.010 & -1.094 & 0.275 \\
Surgery & 13.679 & 4.088 & 3.345 & 0.0010 & 0.082 & 0.037 & 2.224 & 0.027 \\
Surgery & -0.977 & 7.432 & -2.262 & 0.0249 & -0.009 & 0.003 & -2.318 & 0.022 \\
* Month & & & & & & & & \\
\hline
\end{tabular}

group and the subgroup $2, \Omega-\{\mathrm{B}, \mathrm{E}, \mathrm{I}\}$. We also note that no difference between normal group and the subgroup $1,\{\mathrm{~B}, \mathrm{E}, \mathrm{I}\}$. In addition, we find no significant difference between without treatment and with treatment groups. However, we see a significant difference between before surgery and after surgery groups. The growth curve of Protein 2 between before surgery and after surgery groups has changed as time elapses. These test results are given in Table 4 .

Furthermore, we also fit the LME to compare each of three subgroups with the normal group. However, there is no evidence of the difference between the normal and each of these subgroups due to small amount of data for each subgroup (results not shown).

Table 4: Test results fitting a linear mixed effect model which included for covariates the subgroup indicator, the time ( in terms of month) interval, the surgery indicator, the treatment indicator, and the interaction between the time interval and the surgery effect. SG $i$ is a notation for subgroup $i, i=1,2$ and $\mathrm{N}$ stands for the normal group.

\begin{tabular}{|c|cccc||cccc|}
\hline Covariate & \multicolumn{3}{|c||}{ Protein 1 } & \multicolumn{3}{c|}{ Protein 2 } \\
\hline & Mean & S.E & t stat & p-value & Mean & S.E & t stat & p-value \\
SG1 & 5.823 & 3.032 & 1.920 & 0.056 & -0.011 & 0.044 & -0.261 & 0.794 \\
vs SG2 & & & & & & & & \\
SG1 vs N & -1.161 & 1.514 & -0.766 & 0.444 & 0.012 & 0.013 & 0.894 & 0.372 \\
SG2 vs N & -6.985 & 2.529 & -2.761 & 0.0064 & 0.023 & 0.041 & 0.580 & 0.562 \\
Month & -0.825 & 0.111 & -7.385 & 0.0001 & 0.009 & 0.004 & 2.567 & 0.011 \\
Treat & -5.600 & 1.172 & -4.777 & 0.0001 & -0.011 & 0.010 & -1.115 & 0.266 \\
Surgery & 5.941 & 1.890 & 3.142 & 0.0020 & 0.072 & 0.038 & 1.862 & 0.047 \\
Surgery & -0.977 & 0.432 & -2.262 & 0.0251 & -0.009 & 0.003 & -2.290 & 0.023 \\
*month & & & & & & & & \\
\hline
\end{tabular}




\subsection{Cutoff value between normal and disease groups.}

For a fixed type I error rate we calculate the cut off value using the linear mixed effect model. We compare our method with the classical method using the leave-one-out cross valdiation. For each step in the leave-one-out cross validation we omit one patient and calculate the cut off value with remaining patients. We then calculate the sensitivity. We performed this procedure for each patient. The means of cut off values of the cross validation on the each method for three levels of type I error and theirs correspoding sensitivities are given in Tables 5 and 6, respectively Protein 1 and Proten 2. We recall that the growth curve of before surgery group is significantly different from that of after surgery group. In addition we note that the evidence of Status $\{B, E, I\}$ in disease group is not different from normal group. Therefore, the false positive probability which is conditional probability that the test is positive given that the subject does have the disease, were affected by these effects.

Table 5: Means of cut off values for three confidence levels and their corresponding sensitivities of Protein 1 based on a leave-one out cross validation on the classical method and the linear mixed effect model (LME). We used the same type I error rate $(\alpha)$ for each of two methods.

\begin{tabular}{|c|cc|cc|}
\hline Confidence level(\%) & Classical method & & LME & \\
\hline $100^{*}(1-\alpha)$ & Cut off value & Sensitivity & Cut off value & Sensitivity \\
\hline 95 & 51.35 & 0.625 & 53.84 & 0.650 \\
99 & 53.40 & 0.613 & 55.36 & 0.650 \\
99.9 & 54.87 & 0.608 & 57.18 & 0.625 \\
\hline
\end{tabular}

Table 6: Means of cut off values for three confidence levels and their corresponding sensitivities of Protein 2 based on a leave-one out cross validation on the classical method and the linear mixed effect model (LME). We used the same type I error rate $(\alpha)$ for each of two methods.

\begin{tabular}{|c|cc|cc|}
\hline Confidence level(\%) & Classical method & & LME & \\
\hline $100^{*}(1-\alpha)$ & Cut off value & Sensitivity & Cut off value & Sensitivity \\
\hline 95 & 0.657 & 0.700 & 0.710 & 0.758 \\
99 & 0.671 & 0.663 & 0.724 & 0.750 \\
99.9 & 0.688 & 0.650 & 0.740 & 0.725 \\
\hline
\end{tabular}

\section{Discussion}

We has proposed a statistical method of determining a cut off value between normal and disease groups when observations are not independent using linear mixed effect model. This method can be applied to longditudinal data or time course experiemt where the independent assumption of observation does not hold. 
We restrict ourselves study on the linear model which might not detect nonlinear effect. It would be more interest if we could model to catch both linear and nonlinear effects by adding nonlinear term in the model (1). It will be more useful to fit this model without the normality assumption. These aspects of the study are left for the further research.

Figure 3 indicates that there may be two cluster data points of Protien 2. However, we did not particularly attempt to model these two cluster data points, since there was no clinical justfication of observing two clusters of Protein 2 values.

\section{Acknowledgement}

Byung Soo Kim's research was supported by a grant of the Korea Health 21 R\&D Project, Ministry of Health \& Welfare, Republic of Korea (02-PJ1-PG3-10411-00-03). Hyun Cheol Chung's research was supported by the Korea Science and Engineering Fund through the Cancer Metastasis Research Center at Yonsei University.

\section{References}

Laird, N.M. and Ware, J.H. (1982). Random-Effects Models for Longitudinal Data, Biometrics, 38, 963-974.

Lindstrom, M.J. and Bates, D.M. (1988). Newton-Raphson and EM Algorithms for Linear Mixed-Effects Models for Repeated-Measures Data, Journal of the American Statistical Association, 83, 1014-1022.

Pinheiro, J. C. and Bates, D. M. (2000). Mixed-Effect Models in $S$ and S-PLUS, Springer-Verlag, New York.

Rha, S. Y., Yang, W. I., Gong, S. J., Kim, J. J., Kim B. S. and Chung, H. C. (2000). Correlation of Tissue and Blood Plasminogen Activation System in Breast Cancer, Cancer Letters, 150, 137-145.

Received October 18, 2003

Revised April 20, 2004 